\title{
CAUCHY PROBLEM FOR NONLINEAR HYPERBOLIC SYSTEMS OF PARTIAL DIFFERENTIAL EQUATIONS
}

\author{
VICTORIA YASINOVSKAYA
}

\begin{abstract}
We proved the sharp Sobolev estimate for Cauchy data for the general type of hyperbolic systems of nonlinear partial differential equations, which leads to a local existence and uniqueness theorem for solutions of the Cauchy problem in Sobolev spaces.
\end{abstract}

0. Introduction. This paper gives an analysis of the local solvability of the Cauchy problem for hyperbolic systems of nonlinear partial differential equations of the general type:

$$
\vec{e}\left(\vec{x}, D^{m} \vec{u}(\vec{x})\right)=0, \quad D_{x_{0}}^{j} \vec{u}\left(x_{0}=0\right)=\vec{w}_{j}, \quad j=0, \ldots, m-1,
$$

where $x \in \mathbf{R} \times \mathbf{R}^{N}, D^{m} \vec{u}=\left\{D^{\alpha} \vec{u}|| \alpha \mid \leq m\right\}, \vec{w}_{j} \in H_{\mathrm{loc}}^{M-j+1}\left(\mathbf{R}^{N}\right), \vec{e}=\left(e_{1}, \ldots, e_{n}\right)$, and $\vec{u}=\left(u_{1}, \ldots, u_{n}\right)$. In the scalar case it has been proven by P. A. Dionne [4] that for any integer $M>N / 2+1+m$ there exists a unique solution of $(*)$ in $L^{\infty}\left([-T, T], H_{\text {loc }}^{M+N / 2+1}\left(\mathbf{R}^{N}\right)\right)$ for $T$ small enough. In Hughes, Kato, and Marsden [6] it is shown that local solutions for symmetric hyperbolic first order differential equations exist for $M>N / 2+1$.

Bona and Scott have demonstrated in [1] that for $s \geq 2$ the initial value problem for the Korteweg-de Vries equation

$$
u_{t}+u u_{x}+u_{x x x}=0, \quad t>0, \quad u(x, 0)=g(x) \in H^{s}(\mathbf{R})
$$

has a unique solution $u \in C_{0}\left([0, \infty), H^{s}(\mathbf{R})\right)$.

All these results imply that for $M>N / 2+1$ the general Cauchy problem (*) may have a unique solution. Indeed, our major result is

THEOREM 4. For $M>N / 2+1$ the Cauchy problem (*) for hyperbolic nonlinear systems has a unique local solution $\vec{u}$, such that

$$
\frac{\partial^{j}}{\partial t^{j}} \vec{u} \in C\left([-T, T], H_{\mathrm{loc}}^{M-j}\left(\mathbf{R}^{N}\right)\right) \cap L^{\infty}\left([-T, T], H_{\mathrm{loc}}^{M-j+1}\left(\mathbf{R}^{N}\right)\right), \quad j=0, \ldots, m,
$$

if $T$ is small enough, provided

(a)

$$
\vec{e}(\vec{x}, \vec{y}) \in C^{\infty}(\mathbf{R} \times \mathbf{R}^{N}, \underbrace{\mathbf{R}^{r} \times \cdots \times \mathbf{R}^{r}}_{n \text { times }})
$$

where

$$
\vec{y}=D^{m} \vec{u}, \quad r=\left(\begin{array}{c}
m+N+1 \\
N+1
\end{array}\right)
$$

Received by the editors May 2, 1983.

1980 Mathematics Subject Classification. Primary 35L75. 
and

(b)

$$
\text { the matrix } E=\left(\partial e_{i} / \partial y_{j \alpha}\right), \quad 1 \leq i, j \leq n,
$$

is invertible for $\alpha_{0}=m$, where $y_{j \alpha}=D^{\alpha} u_{j}$.

This result is sharp since, as was proved by DiPerna [5] in a much more general context, a continuous (with respect to $t$ ) solution to the Cauchy problem $u_{t}+u u_{x}=$ $0, u(0)=g$ does not exist if $g \in H^{\sigma}(R), \frac{1}{2}<\sigma \leq \frac{3}{2}$.

Establishing Theorem 4 required the following steps. In §II we construct a quasilinear system equivalent to $(*)$, applying the method of $[\mathbf{4}]$, generalized for systems. Then in $\S I I I$ we further reduce it to a first order system which is solved in $§ I V$, where we apply an iterative method and derive energy estimates in order to prove the existence and uniqueness of a solution.

The essential tools that made evaluation of $M$ possible were the techniques of pseudodifferential operators with $H^{M}$ coefficients which were suggested by M. E. Taylor in [7] and paradifferential operators introduced by J. M. Bony in [2].

We consider the Cauchy problem for an $n \times n$ system:

$$
\begin{aligned}
(*) \quad \vec{e}\left(x, D^{m} \vec{u}(x)\right)=0, & x=\left(x_{0}, \ldots, x_{N}\right) \in \mathbf{R}^{N+1}, \\
& \vec{e}, \vec{u} \text { take values in } \mathbf{R}^{n}, \\
D_{0}^{i} u_{j}\left(x_{0}=0\right)=w_{j i}, & i=0, \ldots, m-1, j=1, \ldots, n,
\end{aligned}
$$

with the following.

I. Hypotheses and notation. We denote

$$
\begin{aligned}
& D_{i}=\frac{\partial}{\partial x_{i}}, \quad D^{\alpha}=D_{0}^{\alpha_{0}} D_{1}^{\alpha_{1}} \ldots D_{N}^{\alpha_{N}}, \\
& D_{i}^{\alpha_{i}}=\left(\frac{\partial}{\partial x_{i}}\right)^{\alpha_{i}}, \quad i=0,1, \ldots, N, \\
& D^{m} u_{j}=\left\{D^{\alpha} u_{j}|0 \leq| \alpha|=| \alpha_{0}+\alpha_{1}+\cdots+\alpha_{N} \mid \leq m\right\}, \quad j=1, \ldots, n .
\end{aligned}
$$

Note that for each $j, D^{m} u_{j}$ can be considered as a function taking values in $\mathbf{R}^{r}$, where

$$
r=\left(\begin{array}{c}
m+N+1 \\
N+1
\end{array}\right)=\frac{(m+N+1) !}{(N+1) ! m !} .
$$

Let $y_{i}=D^{m} u_{i}$ for $i=1, \ldots, n$. Then $y_{i}=\left(y_{i_{1}}, \ldots, y_{i_{r}}\right), y_{i \alpha}=D^{\alpha} u_{i}$. Then we can rewrite the system $(*)$ as

$$
\begin{array}{ll}
\vec{e}(x, y(x))=0, & \text { where } y=\left(y_{1}, \ldots, y_{n}\right) \text { takes } \\
& \text { values in } \underbrace{\mathbf{R}^{r} \times \cdots \times \mathbf{R}^{r}}_{n \text { times }} .
\end{array}
$$

Let $e_{k y_{l \alpha}}$ be the partial derivative of $e_{k}$ by $y_{l \alpha}, k=1, \ldots, n ; l=1, \ldots, n ;|\alpha| \leq m$. Define $e_{k x_{i}}(x, y)$ by the equation

$$
\frac{\partial}{\partial x_{i}} e_{k}(x, y(x))=e_{k x_{i}}(x, y(x))+\sum_{l=1}^{n} \sum_{|\alpha|=0}^{m} e_{k y_{l \alpha}} \frac{\partial y_{l \alpha}}{\partial x_{i}} .
$$


Since we are interested in local properties of the solution of (1), let

We suppose

$$
(x, y) \in \mathbf{R} \times \mathbf{T}^{N} \times \underbrace{\mathbf{R}^{r} \times \cdots \times \mathbf{R}^{r}}_{n \text { times }}
$$

(a) $e_{k}(x, y) \in C^{\infty}(X, Y), k=1, \ldots, n$, where $X=\mathbf{R} \times \mathbf{T}^{n}$ and $Y=\underbrace{\mathbf{R}^{r} \times \cdots \times \mathbf{R}^{r}}_{n \text { times }}$.

(b) The matrix

$$
E=\left(\begin{array}{cccc}
e_{1 y_{1 \alpha}} & e_{1 y_{2 \alpha}} & \cdots & e_{1 y_{n \alpha}} \\
e_{2 y_{1 \alpha}} & e_{2 y_{2 \alpha}} & \cdots & e_{2 y_{n \alpha}} \\
& \vdots & & \\
e_{n y_{1 \alpha}} & e_{n y_{2 \alpha}} & \cdots & e_{n y_{n \alpha}}
\end{array}\right),
$$

where $\alpha=(m, 0, \ldots, 0)$, i.e. for $\alpha_{0}=m$, is invertible.

(c) The hyperbolicity condition is satisfied, i.e.

$$
\operatorname{det}\left[\sum_{k=0}^{m}(i \tau)^{m-k} \tilde{A}_{k}\right]
$$

has $m$ purely imaginary, distinct roots $i \tau_{j}(\vec{x}, \vec{\xi}), j=1, \ldots, m$, where

$$
\tilde{A}_{k}=\sum_{\substack{|\alpha|=m \\
\alpha_{0}=m-k}}\left(\begin{array}{cccc}
e_{1 y_{1 \alpha}} & e_{1 y_{2 \alpha}} & \cdots & e_{1 y_{n \alpha}} \\
& \vdots & & \\
e_{n y_{1 \alpha}} & e_{n y_{2 \alpha}} & \cdots & e_{n y_{n \alpha}}
\end{array}\right) \xi^{\left(\alpha_{1}, \ldots, \alpha_{N}\right)} .
$$

(d) $w_{i j} \in H^{M-j+1}\left(\mathbf{T}^{N}\right)$, for $i=1, \ldots, n, j=0, \ldots, m-1$, with $M \in \mathbf{R}$ to be determined.

II. Construction of a quasilinear system. We want to construct a Cauchy problem for a quasilinear hyperbolic system equivalent to (1). We use the method of $[\mathbf{4}]$, generalized for systems. Let

$$
\begin{aligned}
A(x, y, D) & =\sum_{|\alpha|=0}^{m} A_{\alpha}(x, y, D) \\
& =\sum_{|\alpha|=0}^{m}\left(\begin{array}{cccc}
e_{1 y_{1 \alpha}} & e_{1 y_{2 \alpha}} & \cdots & e_{1 y_{n \alpha}} \\
e_{2 y_{1 \alpha}} & e_{2 y_{2 \alpha}} & \cdots & e_{2 y_{n \alpha}} \\
& \vdots & & \\
e_{n y_{1 \alpha}} & e_{n y_{2 \alpha}} & \cdots & e_{n y_{n \alpha}}
\end{array}\right) D^{\alpha},
\end{aligned}
$$

$$
\begin{aligned}
B(x, y) & =\left(\vec{b}_{-1}(x, y), \ldots, \vec{b}_{N}(x, y)\right) \\
& =\left(\begin{array}{ccccc}
e_{10}, & -e_{1 x_{0}}, & -e_{1 x_{1}}, & \cdots, & -e_{1 x_{N}} \\
e_{20}, & -e_{2 x_{0}}, & -e_{2 x_{1}}, & \cdots, & -e_{2 x_{N}} \\
\vdots & & & \\
e_{n 0}, & -e_{n x_{0}}, & -e_{n x_{1}}, & \cdots, & -e_{n x_{N}}
\end{array}\right),
\end{aligned}
$$


where

$$
e_{k 0}(x, y)=\sum_{|\alpha|=0}^{m} \sum_{i=1}^{n} e_{k y_{i \alpha}} y_{i \alpha}-e_{k}, \quad k=1, \ldots, n .
$$

Let $\alpha=\beta(\alpha)+\gamma(\alpha)$, where $\beta(\alpha)=\left(\beta_{0}, \beta_{1}, \ldots, \beta_{N}\right)$ and $\gamma(\alpha)=\left(\gamma_{0}, \gamma_{1}, \ldots, \gamma_{N}\right)$, such that

$$
\begin{aligned}
& |\beta(\alpha)| \leq m-1, \\
& |\gamma(\alpha)|=0 \text { if }|\alpha| \leq m-1, \\
& |\gamma(\alpha)|=1 \text { if }|\alpha|=m,
\end{aligned}
$$

and for each $\gamma(\alpha)$ define $k(\alpha)$ as follows:

if $|\alpha|=m$, then $\gamma(\alpha)=\left(0, \ldots, 0, \gamma_{k(\alpha)}, 0, \ldots, 0\right)$,

if $|\alpha| \leq m-1$, then $k(\alpha)=-1$.

Let

$$
\begin{aligned}
U(x)= & \left(U_{i k}(x)\right)=\left(\vec{U}_{-1}(x), \ldots, \vec{U}_{N}(x)\right) \\
= & \left(\begin{array}{ccccc}
u_{1} & u_{1 x_{0}} & u_{1 x_{1}} & \cdots & u_{1 x_{N}} \\
u_{2} & u_{2 x_{0}} & u_{2 x_{1}} & \cdots & u_{2 x_{N}} \\
& & \vdots & & \\
u_{n} & u_{n x_{0}} & u_{n x_{1}} & & u_{n x_{N}}
\end{array}\right), \\
&
\end{aligned}
$$

and

$$
\begin{aligned}
& W_{j}\left(x_{1}, \ldots, x_{N}\right)=\left(\vec{W}_{-1 j}, \ldots, \vec{W}_{N j}\right) \\
& =\left(\begin{array}{ccccc}
w_{1 j} & w_{1 j+1} & D_{x_{1}} w_{1 j} & \cdots & D_{x_{N}} w_{1 j} \\
w_{2 j} & w_{2 j+1} & D_{x_{1}} w_{2 j} & \cdots & D_{x_{N}} w_{2 j} \\
& & \vdots & & \\
w_{n j} & w_{n j+1} & D_{x_{1}} w_{n j} & \cdots & D_{x_{N}} w_{n j}
\end{array}\right), \\
& j=0, \ldots, m-1,
\end{aligned}
$$

be vector valued functions in $\mathbf{R} \times \mathbf{T}^{N}$ and $\mathbf{T}^{N}$ respectively. (Here functions $w_{1 m}=$ $D_{0}^{m} u_{1}\left(x_{0}=0\right), \ldots, w_{n m}=D_{0}^{m} u_{n}\left(x_{0}=0\right)$, which appear in (8) when $j=m-1$, are determined by (1).)

Now consider the Cauchy problem for the following quasilinear system of order $m$ :

$$
\left\{\begin{array}{l}
A\left(x, D^{\beta(\alpha)} \vec{U}_{k(\alpha)}, D\right) U=B\left(x, D^{\beta(\alpha)} \vec{U}_{k(\alpha)}\right), \\
D_{0}^{j} U\left(x_{0}=0\right)=W_{j}, \quad \text { where } j=0, \ldots, m-1 ; W_{j} \in H^{M-j}\left(\mathbf{T}^{N}\right) .
\end{array}\right.
$$

Here the components, $U_{i k(\alpha)}$, of $\vec{U}_{k(\alpha)}$ are entries of the column $k(\alpha)$ in the matrix $(7), k(\alpha)=-1,0,1, \ldots, N$, and $D^{\beta(\alpha)} U_{i k(\alpha)}$ are substituted for $y_{i \alpha}$ in (4) and (5), $i=1, \ldots, n$.

LEMMA 1. Cauchy problems (1) and (9) are equivalent.

PROOF. The $n(N+2)$ equations in (9) can be separated into two different types. 
First type:

$$
\sum_{|\alpha|=0}^{m} \sum_{i=1}^{n} e_{l y_{i \alpha}}\left(x, D^{\beta(\alpha)} U_{k(\alpha)}\right) D^{\alpha} u_{i}=e_{l 0}\left(x, D^{\beta(\alpha)} U_{k(\alpha)}\right)
$$

for $l=1, \ldots, n$, which is

$$
\begin{aligned}
& \sum_{|\alpha|=0}^{m-1} \sum_{i=1}^{n} e_{l y_{i \alpha}}\left(x, D^{\alpha} \vec{u}\right) y_{i \alpha}+\sum_{|\alpha|=m} \sum_{i=1}^{n} e_{l y_{i \alpha}}\left(x, D^{\beta(\alpha)} U_{k(\alpha)}\right) D^{\alpha} u_{i} \\
& =\sum_{|\alpha|=0}^{m-1} \sum_{i=1}^{n} e_{l y_{i \alpha}} y_{i \alpha}+\sum_{|\alpha|=m} \sum_{i=1}^{n} e_{l y_{i \alpha}}\left(x, D^{\beta(\alpha)} U_{k(\alpha)}\right) D^{\beta(\alpha)} U_{k(\alpha)}-e_{l} .
\end{aligned}
$$

Here the first term on the left is equal to the first term on the right side of the equation. By definition of $\beta(\alpha)$ and $k(\alpha)$ the second term on the left and right sides are also equal.

Second type:

$$
\sum_{|\alpha|=0}^{m} \sum_{i=1}^{n} e_{l y_{i \alpha}}\left(x, D^{\beta(\alpha)} U_{k(\alpha)}\right) D^{\alpha} u_{i x_{j}}=-e_{l x_{j}}\left(x, D^{\alpha} \vec{u}\right)
$$

where $l=1, \ldots, n ; j=0, \ldots, N$. We rewrite the last equation as

$$
\begin{aligned}
& \sum_{|\alpha|=0}^{m-1} \sum_{i=1}^{n} e_{l y_{i \alpha}}\left(x, D^{\alpha} \vec{u}\right) D^{\alpha} u_{i x_{j}} \\
& \quad+\sum_{|\alpha|=m} \sum_{i=1}^{n} e_{l y_{i \alpha}}\left(x, D^{\beta(\alpha)} \vec{U}_{k(\alpha)}\right) D^{\alpha} u_{i x_{j}} \\
& \quad=-e_{l x_{j}}\left(x, D^{\alpha} \vec{u}\right) .
\end{aligned}
$$

Since $D^{\beta(\alpha)} \vec{U}_{k(\alpha)}=D^{\alpha} \vec{u}$ for $|\alpha|=m$, where $\vec{u}$ is a column $\left(u_{1}, \ldots, u_{n}\right)^{t}$, we use (3) to see that equation (11) means

$$
\frac{\partial}{\partial x_{i}} e_{l}\left(x, D^{\alpha} \vec{u}\right)=0 .
$$

We conclude that (10) and (11) are equivalent to (1). The second condition in (9) is equivalent to hypothesis $\mathrm{I}(\mathrm{d})$. Q.E.D.

III. Reduction to a first order system. In order to solve (9) we reduce it to a first order system as follows. Let us change notations: $\left(x_{0}, x_{1}, \ldots, x_{N}\right)=(t, x)$. Multiply both sides of (9) by $E^{-1}$ on the left (see hypothesis I(b)) and rewrite it in the form

$$
\begin{gathered}
{\left[\frac{\partial^{m}}{\partial t^{m}} I_{n}-\sum_{i=0}^{m-1} A_{m-i}\left(t, x, D^{\beta(\alpha)} U_{k(\alpha)}, D_{x}\right) \frac{\partial^{i}}{\partial t^{i}}\right] U} \\
=f\left(t, x, D^{\beta(\alpha)} U_{k(\alpha)}\right),
\end{gathered}
$$

where $A_{m-i}\left(t, x, y, D_{x}\right)$ is a differential operator of order $m-i$ with top order symbol $\tilde{A}_{m-i}$ and

$$
f\left(t, x, D^{\beta(\alpha)} U_{k(\alpha)}\right)=E^{-1} B\left(t, x, D^{\beta(\alpha)} U_{k(\alpha)}\right) .
$$


Since the next step in the transformation of equation (12) will involve a pseudodifferential operator of a certain class (see [7]), we will give the following

DEFinition A. Let $\Omega$ be an open subset of $\mathbf{R}^{N}, m \in \mathbf{R}$. We define the symbol class $S_{1,0}^{m}(\Omega)$ to consist of the set of $p(x, \xi) \in C^{\infty}\left(\Omega \times \mathbf{R}^{N}\right)$ with the property that, for any compact $K \subset \Omega$ and any multi-indices $\alpha, \beta$, there exists a constant $C_{K, \alpha, \beta}$ such that

$$
\left|D_{x}^{\beta} D_{\xi}^{\alpha} p(x, \xi)\right| \leq C_{K, \alpha, \beta}(1+|\xi|)^{m-|\alpha|}
$$

for all $x \in K, \xi \in \mathbf{R}^{N}$. In this case the operator $p(x, D)$ is said to belong to $O P S_{1,0}^{m}(\Omega)$.

If, moreover, there are smooth functions $p_{m-j}(x, \xi)$, homogeneous of degree $m-j$ in $\xi$ for $|\xi| \geq 1$ such that

$$
p(x, \xi) \sim \sum_{j \geq 0} p_{m-j}(x, \xi)
$$

where the asymptotic condition means that

$$
p(x, \xi)-\sum_{j=0}^{M} p_{m-j}(x, \xi) \in S_{1,0}^{m-M-1}(\Omega),
$$

then we say that symbol $p(x, \xi) \in S^{m}(\Omega)$ and the operator $p(x, D) \in O P S^{m}(\Omega)$.

We can now define the concept of a pseudodifferential operator on a manifold $M$.

DEFINITION B. $p(x, D): C_{0}^{\infty}(M) \rightarrow C^{\infty}(M)$ belongs to $O P S_{1,0}^{m}(M)\left(O P S^{m}(M)\right)$ if $p(x, \xi)$ is smooth on $M$ and if for any coordinate neighborhood $U$ in $M$ with $\chi: U \rightarrow O$ a diffeomorphism onto an open subset $O$ of $\mathbf{R}^{N}$, the map of $C_{0}^{\infty}(0)$ into $C^{\infty}(0)$ given by $u \mapsto p(x, D)(u \circ \chi) \circ \chi^{-1}$ belongs to $O P S_{1,0}^{m}(0)\left(O P S^{m}(0)\right)$.

Now let $U^{j}=(\partial / \partial t)^{j-1} \Lambda^{m-j} U, j=1, \ldots, m$, and $\Lambda=(1-\Delta)^{1 / 2} \in O P S^{1}$. Then (12) is equivalent to

$$
\frac{\partial}{\partial t}\left(\begin{array}{c}
U^{1} \\
\cdot \\
\cdot \\
\cdot \\
U^{m}
\end{array}\right)=\left(\begin{array}{ccccc}
O_{n} & \Lambda_{n} & O_{n} & \cdots & O_{n} \\
& O_{n} & \Lambda_{n} & & \vdots \\
& & & \ddots & \\
& & & & \Lambda_{n} \\
\left(b_{1}\right) & \left(b_{2}\right) & \left(b_{3}\right) & \cdots & \left(b_{m}\right)
\end{array}\right)\left(\begin{array}{c}
U^{1} \\
\cdot \\
\cdot \\
\cdot \\
U^{m}
\end{array}\right)+\left(\begin{array}{c}
O_{n} \\
\cdot \\
\cdot \\
\cdot \\
O_{n} \\
f
\end{array}\right)
$$

where $b_{j}=A_{m-j+1}\left(t, x, P_{j 1}\left(U^{1}, \ldots, U^{m}\right)^{t}, \ldots, P_{j \nu}\left(U^{1}, \ldots, U^{m}\right)^{t}, D_{x}\right) \Lambda^{j-m}$ with $P_{j \mu} \in O P S^{s}, s \leq 0$.

Each $U^{j}$ in $(13)$ is an $n$-vector, hence

$$
\left(\begin{array}{c}
U^{1} \\
\cdot \\
\cdot \\
U^{m}
\end{array}\right)
$$

is an $m n$-vector. Each entry in the matrix of equation (13) is an $n \times n$ matrix, for example, $\Lambda_{n}=\Lambda I_{n}, O_{n}=0 I_{n}$, so (13) is an $(m \cdot n) \times(m \cdot n)$ system. Changing 
notation, calling the column vector $\left(U^{1}, \ldots, U^{m}\right)^{t} U$ and the matrix in (13) $K$, write (13) with the initial data as

$$
\begin{aligned}
\frac{\partial}{\partial t} U & =K\left(t, x, P_{1} U, \ldots, P_{l} U, D_{x}\right) U+f\left(t, x, P_{1} U, \ldots, P_{l} U\right), \\
U(0) & =g
\end{aligned}
$$

where

$$
g=\left(\begin{array}{c}
g_{1} \\
\vdots \\
g_{m}
\end{array}\right)=\left(\begin{array}{c}
\Lambda^{m-1} W_{0} \\
\vdots \\
W_{m-1}
\end{array}\right), \text { i.e. } g_{j}=\Lambda^{m-j} W_{j-1},
$$

and the $W_{j}$ 's are given by (8), and where $P_{j} \in O P S^{0}$ and $K\left(t, x, U_{1}, \ldots, U_{l}, \xi\right) \in$ $S^{1}$.

IV. Solution of the first order hyperbolic system. By hypothesis I(c) system (14) is hyperbolic, and in order to solve it we will use an iterative method as follows. Given $U$ on $\mathbf{R} \times \mathbf{T}^{N}$ with $U(0)=g$, we define $F U=V$ to be the solution to the system

$$
\begin{aligned}
\frac{\partial}{\partial t} V & =K\left(t, x, P_{1} U, \ldots, P_{l} U, D_{x}\right) V+f\left(t, x, P_{1} U, \ldots, P_{l} U\right), \\
V(0) & =g
\end{aligned}
$$

and find a fixed point of $F$, i.e. a function $U$ such that $F U=U$ on $(-T, T) \times \mathbf{T}^{N}$. In order to treat this problem, it will be necessary to introduce pseudodifferential operators with less than $C^{\infty}$ symbols (see [7]) and paradifferential operators (see [2]).

Definition 1. We say $p(x, \xi) \in H^{M} S_{1,0}^{m}\left(\mathbf{T}^{N}\right)$ and $p(x, D) \in O P H^{M} S_{1,0}^{m}\left(\mathbf{T}^{N}\right)$ provided

$$
\left\|D_{\xi}^{\alpha} p(x, \xi)\right\|_{H^{M}\left(\mathbf{T}^{N}\right)} \leq C(1+|\xi|)^{m-|\alpha|} \text { for }|\alpha| \leq M .
$$

If, morover, there are functions $p_{m-j}(x, \xi) \in H^{M} S_{1,0}^{m-j}\left(\mathbf{T}^{N}\right)$, homogeneous of degree $m-j$ in $\xi$ for $|\xi| \geq 1$ such that

$$
p(x, \xi) \sim \sum_{j \geq 0} p_{m-j}(x, \xi)
$$

then we say that $p(x, \xi) \in H^{M} S^{m}\left(\mathbf{T}^{N}\right)$ and $p(x, D) \in O P H^{M} S^{m}\left(\mathbf{T}^{N}\right)$.

DEFinition 2. Let $\chi(\theta, \eta)$ be a $C^{\infty}$ function defined on $\mathbf{R}^{N} \times \mathbf{R}^{N} \backslash O$, homogeneous of degree 0 , such that for some small $\varepsilon_{1}, \varepsilon_{2}, 0 \leq \varepsilon_{1}<\varepsilon_{2}, \chi(\theta, \eta)=1$ for $|\theta| \leq \varepsilon_{1}|\eta|, \chi(\theta, \eta)=0$ for $|\theta| \geq \varepsilon_{2}|\eta|$, and let $s(\eta)$ be a $C^{\infty}$ function from $\mathbf{R}^{N}$ to $\mathbf{R}$ which is 0 in some neighborhood of $O$ and 1 outside of some compact subset of $\mathbf{R}^{N}$. Let $l(x, \xi)$ be a function homogeneous of degree $m$ in $\xi, C^{\infty}$ in $\xi$ for $\xi \neq 0$, with compact support with respect to $x$, and $C^{\rho}$ in $x$ for $\rho$ noninteger. Define the operator $T_{l}$ on the space of distributions, $D^{\prime}$, as

$$
\left(T_{l} u\right)^{-}(\xi)=\int \chi(\xi-\eta, \eta) \hat{l}(\xi-\eta, \eta) s(\eta) \hat{u}(\eta) d \eta
$$

where $\hat{l}(\theta, \xi)$ is the Fourier transform of $l(x, \xi)$ with respect to the first variable. 
Definition 3. (a) For any $\Omega \subset \mathbf{R}^{N}, m \in \mathbf{R}, \rho>0$ and noninteger, we define $\Sigma_{\boldsymbol{\rho}}^{m}(\Omega)$ as the set of all functions $l(x, \xi)$ in $\Omega \times\left(\mathbf{R}^{N} \backslash O\right)$ such that

$$
l(x, \xi)=l_{m}(x, \xi)+l_{m-1}(x, \xi)+\cdots+l_{m-[\rho]}(x, \xi),
$$

where $l_{m-k}(x, \xi)$ is homogeneous of degree $m-k$ in $\xi$, is $C^{\infty}$ in $\xi$, and $C_{\text {loc }}^{\rho-k}$ in $x$.

(b) If $l^{(i)} \in \Sigma_{\rho}^{m_{i}}, i=1,2$, we define $l^{1} \# l^{2} \in \Sigma_{\rho}^{m_{1}+m_{2}}$ as

$$
l^{1} \# l^{2}=\sum_{|\alpha|+k_{1}+k_{2} \leq[\rho]} \sum_{\frac{1}{\alpha !}} D_{\xi}^{\alpha} l_{m_{1}-k_{1}}^{1} D_{x}^{\alpha} l_{m_{2}-k_{2}}^{2} .
$$

(c) If $l \in \Sigma_{\rho}^{m}$, we define $l^{*} \in \Sigma_{\rho}^{m}$ as

$$
l^{*}=\sum_{|\alpha|+k \leq[\rho]} \sum_{\frac{1}{\alpha !}} D_{\xi}^{\alpha} D_{x}^{\alpha} \bar{l}_{m-k} .
$$

Definition 4. Let $\Omega$ be an open subset of $\mathbf{R}^{N}$ and let $L$ be a linear transformation in $D^{\prime}(\Omega)$, which is properly supported, i.e. for any compact $K$ in $\Omega$ there is a compact $\hat{K}$ in $\Omega$ such that

$$
\operatorname{supp} u \subset K \Rightarrow \operatorname{supp} L u \subset \hat{K}
$$

and

$$
(\operatorname{supp} u) \cap \hat{K}=\varnothing \Rightarrow \operatorname{supp} L u \cap K=\varnothing .
$$

Then we call $L$ a paradifferential operator of order $m$ and of class $C^{\rho}$ in $\Omega$ and write $L \in O P\left(\Sigma_{\rho}^{m}\right)(\Omega)$ if there exists $l \in \Sigma_{\rho}^{m}(\Omega)$ such that for any compact $K \subset \Omega$ and any $\chi \in C_{0}^{\infty}(\Omega)$ which is equal to 1 in a neighborhood of $K$, the operator $L-\chi T_{\chi \cdot l}$ is a continuous map of elements of $H^{s}$ with support in $K$ to $H^{s-m+\rho}$. In this case $l$ is called a symbol of $L$.

The proofs of the following results are given in [2].

THEOREM 1. (a) If $L \in O P\left(\Sigma_{\rho}^{m}\right)(\Omega)$, then $L: H_{\mathrm{loc}}^{s}(\Omega) \rightarrow H_{\mathrm{loc}}^{s-m}(\Omega)$.

(b) If $L \in O P\left(\Sigma_{\rho}^{m}\right)(\Omega)$, there exists a unique symbol l of $L$, i.e. $\sigma(L)=l$.

(c) If $L^{j} \in O P\left(\Sigma_{\rho}^{m_{j}}\right)(\Omega), j=1,2$, then $L^{1} L^{2} \in O P\left(\Sigma_{\rho}^{m_{1}+m_{2}}\right)(\Omega)$ and $\sigma\left(L^{1} \cdot L^{2}\right)=\sigma\left(L^{1}\right) \# \sigma\left(L^{2}\right)$.

(d) If $L \in O P\left(\Sigma_{\rho}^{m}\right)(\Omega)$, then $\sigma\left(L^{*}\right)=(\sigma(L))^{*}$, where $L^{*}$ is the adjoint of $L$.

(e) If $L$ is a classical pseudodifferential operator of order $m$, properly supported in $\Omega$, with symbol

$$
l(x, \xi) \sim \sum_{j \geq 0} l_{m-j}(x, \xi),
$$

then for any $\rho>0, L \in O P\left(\sum_{\rho}^{m}\right)$ with symbol $\sigma(L)=\sum_{0 \leq j \leq[\rho]} l_{m-j}$.

NOTE. If $l(x, \xi)$ is such that $l_{m-j}(x, \xi)$ is in $C^{\gamma-j}$ as a function of $x$, then $L \in O P\left(\Sigma_{\gamma}^{m}\right)$ and $\sigma(L)=\sum_{0 \leq j \leq[\gamma]} l_{m-j}$ where $\gamma$ is not an integer.

To return to our iterative method (15), we assume that $U \in C\left([-T, T], H^{M}\left(\mathbf{T}^{N}\right)\right)$ and $\partial U / \partial t \in C\left([-T, T], H^{M}\left(\mathbf{T}^{N}\right)\right)$ for $M$ to be estimated. Then

$$
K\left(t, x, P_{1} U, \ldots, P_{l} U, D_{x}\right) \in O P H^{M} S_{1,0}^{1}
$$

and in order to solve the quasilinear hyperbolic system

$$
\frac{\partial}{\partial t} V=K V+f, \quad V(0)=g
$$


with $f \in C\left([-T, T], H^{M}\left(\mathbf{T}^{N}\right)\right), g \in H^{M}\left(\mathbf{T}^{N}\right)$, we need to construct what is called a symmetrizer for $K$.

Definition 5. Let $K \in O P S_{1,0}^{1}$. Then a symmetrizer for $(\partial / \partial t)-K$ is a smooth one parameter family of operators $R=R(t) \in O P S^{0}$, such that

$$
R_{0}(t, x, \xi) \text { is a positive definite matrix for }|\xi| \geq 1,
$$

$$
R K+(R K)^{*} \in O P S_{1,0}^{0} .
$$

If such a symmetrizer exists, one says $\partial / \partial t-K$ is symmetrizable.

The proof of the following proposition can be found in [2].

Proposition 1. Any strictly hyperbolic first order system $\partial / \partial t-K$, where $K \in O P S_{1,0}^{1}$, has a symmetrizer $R$ and we have

$$
R\left(t, x, D_{x}\right)=\sum_{j=1}^{k} P_{j}(t, x, D)^{*} P_{j}(t, x, D),
$$

$$
\sigma_{R K}(t, x, \xi)=i \sum_{j=1}^{k} \lambda_{j}(t, x, \xi) P_{j}(t, x, \xi)^{*} P_{j}(t, x, \xi) \bmod S^{0},
$$

where $i \lambda_{\nu}(t, x, \xi)$ are eigenvalues of $K_{1}(t, x, \xi)$, the principal symbol of $K, \lambda_{1}(t, x, \xi)$ $<\lambda_{2}(t, x, \xi)<\cdots<\lambda_{k}(t, x, \xi)$, and $P_{j}(t, x, \xi) \in S^{0}$ are the projections onto the associated eigenspaces of $i \lambda_{j}(t, x, \xi)$ :

$$
P_{j}=\frac{1}{2 \pi i} \int_{\gamma_{j}}\left(\zeta-K_{1}(t, x, \xi)\right)^{-1} d \zeta
$$

where $\gamma_{j}$ is the circle around $\lambda_{j}$ only.

Now we are ready to prove the following result.

Proposition 2. If $M>N / 2+1$, then, given $K \in O P H^{M} S^{1}\left(\mathbf{T}^{N}\right)$, the hyperbolic system

$$
\partial V / \partial t=K V+f, \quad V(0)=g
$$

has a unique solution $V \in C\left([-T, T], H^{M}\left(\mathbf{T}^{N}\right)\right)$ for $g \in H^{M}\left(\mathbf{T}^{N}\right)$ and $f \in$ $C\left([-T, T], H^{M}\left(\mathbf{T}^{N}\right)\right)$. Such a solution satisfies the estimate

$$
\|V(t)\|_{H^{M}}^{2} \leq(1+C|t|)\left[\|g\|_{H^{M}}^{2}+\int_{0}^{t}\|f(\tau)\|_{H^{M}}^{2} d \tau\right], \quad|t| \leq T,
$$

where $C$ depends on finitely many seminorms of $K \in O P H^{M} S^{1}\left(\mathbf{T}^{N}\right), R \in$ $O P H^{M} S^{0}\left(\mathbf{T}^{N}\right), \partial R / \partial t \in O P H^{M} S^{0}\left(\mathbf{T}^{N}\right)$, and $R K+(R K)^{*} \in O P H^{L} S^{0}\left(\mathbf{T}^{N}\right)$ for some $L<M$, and on $N$ but not on the order of the system.

The following inequality from the theory of ordinary differential equations will be useful in the proof of Proposition 2. 

then

Lemma 2 (GRonwall's INEQUality). If $y \in C^{1}$ and $y^{1}(t)+f(t) y \leq g(t)$,

$$
y(t) \leq e^{-\int_{0}^{t} f(\tau) d \tau}\left[y_{0}+\int_{0}^{t} g(\tau) e^{\int_{0}^{\tau} f(\sigma) d \sigma} d \tau\right] .
$$

Proof of Proposition 2. Since $K \in O P H^{M} S^{1}\left(\mathbf{T}^{N}\right)$, it is clear from (19) that the symmetrizer $R \in O P H^{M} S^{0}\left(\mathbf{T}^{N}\right)$. Then we have the following asymptotic expansions of the symbols $K(t, x, \xi) \in H^{M} S^{1}$ and $R(t, x, \xi) \in H^{M} S^{0}$ :

$$
K(t, x, \xi) \sim \sum_{j \geq 0} K_{1-j}(t, x, \xi), \quad R(t, x, \xi) \sim \sum_{j \geq 0} R_{-j}(t, x, \xi)
$$

where $K_{1-j}(t, x, \xi)$ and $R_{-j}(t, x, \xi)$ are homogeneous functions in $\xi$ of degree $1-j$ and $-j$ respectively and are in $H^{M}\left(\mathbf{T}^{N}\right)$ as functions of $x$. Then, by the Sobolev imbedding theorem, $K_{1-j}$ and $R_{-j}$ belong to $C^{M-N / 2}\left(\mathbf{T}^{N}\right)$ and we can apply Theorem 1 to conclude that

$$
K\left(t, x, D_{x}\right) \in O P\left(\Sigma_{M-N / 2}^{1}\right)
$$

with $\sigma(K)=\sum_{0 \leq j \leq[M-N / 2]} K_{1-j}(t, x, \xi)$ and

$$
R\left(t, x, D_{x}\right) \in O P\left(\Sigma_{M-N / 2}^{0}\right)
$$

with $\sigma(R)=\sum_{0 \leq j \leq[M-N / 2]} R_{-j}(t, x, \xi)$ for $M-N / 2$ noninteger.

NOTE. In the case when $M-N / 2$ is an integer we can find some small $\varepsilon>0$, such that $M-N / 2-\varepsilon>1$ (since by hypothesis $M-N / 2>1$ ) and replace $M-N / 2$ by noninteger $M-N / 2-\varepsilon$ for all symbols above.

It follows that $R K \in O P\left(\Sigma_{M-N / 2}^{1}\right)$ with

$$
\sigma(R K)=R_{0} K_{1}+\sum_{1 \leq|\alpha|+j_{1}+j_{2} \leq[M-N / 2]} \sum_{\alpha !} \frac{1}{\alpha !} D_{\xi}^{\alpha} R_{-j_{1}} D_{x}^{\alpha} K_{1-j_{2}}
$$

where for each $|\alpha|, j_{1}$, and $j_{2}$ the corresponding term is homogeneous of degree $1-\left(|\alpha|+j_{1}+j_{2}\right)$ in $\xi$ and belongs to $C^{[M-N / 2]-\left(|\alpha|+j_{1}+j_{2}\right)}\left(\mathbf{T}^{N}\right)$ in $x$. Hence

$$
\begin{aligned}
& \sigma\left((R K)^{*}\right)=\sum_{|\beta|+|\alpha|+j_{1}+j_{2} \leq[M-N / 2]} \sum_{\alpha ! \beta !} D_{\xi}^{\beta} D_{x}^{\beta} \overline{D_{\xi}^{\alpha} R_{-j_{1}} D_{x}^{\alpha} K_{1-j_{2}}} \\
& =R_{0} \bar{K}_{1}+\sum_{1 \leq|\beta|+|\alpha|+j_{1}+j_{2} \leq[M-N / 2]} \sum_{\alpha ! \beta !} D_{\xi}^{\beta} D_{x}^{\beta} D_{\xi}^{\alpha} R_{-j_{1}} D_{x}^{\alpha} \bar{K}_{1-j_{2}}
\end{aligned}
$$

and since $K_{1}(t, x, \xi)$ has purely imaginary distinct eigenvalues for each $(t, x, \xi)$, we have

(22)

$$
\begin{aligned}
\sigma\left(R K+(R K)^{*}\right)= & \sigma(R K)+\sigma\left((R K)^{*}\right) \\
= & \sum_{1 \leq|\alpha|+j_{1}+j_{2} \leq[M-N / 2]} \sum_{1 \leq|\beta|+|\alpha|+j_{1}+j_{2} \leq[M-N / 2]} \frac{1}{\alpha !} D_{\xi}^{\alpha} R_{-j_{1}} D_{x}^{\alpha} K_{1-j_{2}} \\
& +\sum_{1 \leq \beta !} D_{\xi}^{\beta} D_{x}^{\beta} D_{\xi}^{\alpha} R_{-j_{1}} D_{x}^{\alpha} \bar{K}_{1-j_{2}} .
\end{aligned}
$$


Thus $R K+(R K)^{*} \in O P\left(\Sigma_{M-N / 2-1}^{0}\right)$, which justifies the hypothesis that $M>$ $N / 2+1$. Applying Theorem 1 again, we conclude that

$$
\begin{aligned}
& R K+(R K)^{*}: H^{s}\left(\mathbf{T}^{N}\right) \rightarrow H^{s}\left(\mathbf{T}^{N}\right) \text { as well as } \\
& R: H^{s}\left(\mathbf{T}^{N}\right) \rightarrow H^{s}\left(\mathbf{T}^{N}\right), \\
& \frac{\partial}{\partial t} R: H^{s}\left(\mathbf{T}^{N}\right) \rightarrow H^{s}\left(\mathbf{T}^{N}\right), \text { and } \\
& K: H^{s}\left(\mathbf{T}^{N}\right) \rightarrow H^{s-1}\left(\mathbf{T}^{N}\right) \quad \text { for any } s \in \mathbf{R} .
\end{aligned}
$$

Now we can obtain the energy estimate for $V$ solving (21). We write

$$
\begin{aligned}
\frac{d}{d t}(R V, V) & =\left(R V_{t}, V\right)+\left(R V, V_{t}\right)+\left(R_{t} V, V\right) \\
& =(R K V+R f, V)+(R V, K V+f)+\left(R_{t} V, V\right) \\
& =\left(\left(R K+K^{*} R\right) V, V\right)+(R f, V)+(R V, f)+\left(R_{t} V, V\right) \\
& \leq C_{1}\|V\|_{L^{2}}^{2}+C_{2}\|f\|_{L^{2}}^{2} \leq C(R V, V)+C\|f\|_{L^{2}}^{2} .
\end{aligned}
$$

Applying Gronwall's inequality (Lemma 2) to this yields

$$
\|V(t)\|_{L^{2}}^{2} \leq(1+C|t|)\left[\|g\|_{L^{2}}^{2}+\int_{0}^{t}\|f(\tau)\|_{L^{2}}^{2} d \tau\right]
$$

for $t \in[-T, T]$.

More generally, differentiating

$$
\|V(t)\|_{H^{s}\left(\mathbf{T}^{N}\right)}^{2}=\left\|\Lambda^{s} V(t)\right\|_{L^{2}\left(\mathbf{T}^{N}\right)}^{2}
$$

yields

$$
\|V(t)\|_{H^{*}}^{2} \leq(1+C|t|)\left[\|g\|_{H^{s}}^{2}+\int_{0}^{t}\|f(\tau)\|_{H^{s}}^{2} d \tau\right]
$$

for $t \in[-T, T]$, which is bounded in a bounded interval $-T \leq t \leq T$. $C$ is a function of finitely many seminorms of $K \in O P H^{M} S^{1}, R \in O P H^{M} S^{0}, \partial R / \partial t \in$ $O P H^{M} S^{0}$, and $R K+(R K)^{*} \in O P H^{L} S^{0}$ for some $L<M$ which we need not specify, and of $N$. Inequality (26) is valid whenever $u \in H^{1}\left([-T, T], H^{s+1}\left(\mathbf{T}^{N}\right)\right)$, $f \in L^{2}\left([-T, T], H^{s}\left(\mathbf{T}^{N}\right)\right), g \in H^{s+1}\left(\mathbf{T}^{N}\right)$, and (21) is satisfied. We shall obtain the solution of the initial value problem (21) as a limit of solutions to the problem

$$
\frac{\partial}{\partial t} V=K J_{\varepsilon} V+f, \quad V(0)=g
$$

where $J_{\varepsilon}$ is a Friedrich's mollifier on $\mathbf{T}^{N}$ defined as the following:

DEFINITION 6. A Friedrich's mollifier on $\mathbf{T}^{N}$ is a family, $J_{\varepsilon}$, of scalar pseudodifferential operators, $0 \leq \varepsilon \leq 1$, such that

$$
\begin{aligned}
& J_{\varepsilon} \in O P S^{-\infty}\left(\mathbf{T}^{N}\right) \text { for each } \varepsilon \in(0,1], \\
& \left\{J_{\varepsilon}: 0<\varepsilon \leq 1\right\} \text { is a bounded subset of } O P S_{1,0}^{0}\left(\mathbf{T}^{N}\right), \text { and } \\
& J_{\varepsilon} u \rightarrow u \text { in } L^{2}\left(\mathbf{T}^{N}\right) \text { as } \varepsilon \rightarrow 0 \text { for each } u \in L^{2}\left(\mathbf{T}^{N}\right) .
\end{aligned}
$$

To construct a Friedrich's mollifier, we multiply $\varepsilon^{-|\varepsilon \xi|^{2}}$ by a partition of unity for some coordinate patches on $\mathbf{T}^{N}$. 
Now for each $\varepsilon>0, K_{\varepsilon}=K J_{\varepsilon}$ is a continuous linear operator on $H^{s}$. Hence (27) can be considered as a Banach-space valued ordinary differential equation and to solve it we apply the Picard iteration method (see Dieudonné [3]). Thus, given $g \in H^{s+1}\left(\mathbf{T}^{N}\right)$ and $f \in C\left([-T, T], H^{s+1}\left(\mathbf{T}^{N}\right)\right)$, we can solve (27), producing a solution $V_{\varepsilon} \in C^{1}\left([-T, T], H^{s+1}\left(\mathbf{T}^{N}\right)\right)$. Now since $\left\{K_{\varepsilon}: 0<\varepsilon \leq 1\right\}$ is a bounded subset of $O P H^{M} S_{1,0}^{1}\left(\mathbf{T}^{N}\right)$ and $\left\{R K_{\varepsilon}+\left(R K_{\varepsilon}\right)^{*}: 0<\varepsilon \leq 1\right\}$ is a bounded subset of $O P H^{L} S_{1,0}^{0}\left(\mathbf{T}^{N}\right)$ for some $L<M$, we get the estimate (26) for $V_{\varepsilon}$ :

$$
\left\|V_{\varepsilon}\right\|_{H^{s}}^{2} \leq(1+C|t|)\left[\|g\|_{H^{s}}^{2}+\int_{0}^{t}\|f(\tau)\|_{H^{s}}^{2} d \tau\right]
$$

with $C$ independent of $\varepsilon, 0<\varepsilon \leq 1$. Now by this estimate, $\left\{V_{\varepsilon}: 0<\varepsilon \leq\right.$ $1\}$ is a bounded subset of $C\left([-T, T], H^{s}\left(\mathbf{T}^{N}\right)\right)$ given $g \in H^{s+1}\left(\mathbf{T}^{N}\right)$ and $f \in$ $C\left([-T, T], H^{s+1}\left(\mathbf{T}^{N}\right)\right)$. Since $V_{\varepsilon}^{\prime}=K_{\varepsilon} V_{\varepsilon}+f$ and $\left\{K_{\varepsilon}: 0<\varepsilon \leq 1\right\}$ is a bounded subset of $O P H^{M} S_{1,0}^{1}\left(\mathbf{T}^{N}\right)$, it follows that $\left\{V_{\varepsilon}^{\prime}: 0<\varepsilon \leq 1\right\}$ is a bounded subset of $C\left([-T, T], H^{s-1}\left(\mathbf{T}^{N}\right)\right)$. Hence $\left\{V_{\varepsilon}: 0<\varepsilon \leq 1\right\}$ is a bounded subset of $C^{1}\left([-T, T], H^{s-1}\left(\mathbf{T}^{N}\right)\right)$. Furthermore, for each $t_{0} \in[-T, T],\left\{V_{\varepsilon}\left(t_{0}\right): 0<\varepsilon \leq 1\right\}$, being a bounded subset of $H^{s}\left(\mathbf{T}^{N}\right)$, is a relatively compact subset of $H^{s-1}\left(\mathbf{T}^{N}\right)$. Hence, by Ascoli's theorem [3], there is a sequence $\varepsilon_{n} \rightarrow 0$ such that $V_{\varepsilon_{n}}$ converges in $C\left([-T, T], H^{s-1}\left(\mathbf{T}^{N}\right)\right)$ to a limit we call $V$, which satisfies (21) in the sense of distributions.

Now let $g_{j} \in H^{s+4}\left(\mathbf{T}^{N}\right)$ with $g_{j} \rightarrow g$ in $H^{s}\left(\mathbf{T}^{N}\right)$ and let

$$
f_{j} \in C\left([-T, T], H^{s+4}\left(\mathbf{T}^{N}\right)\right),
$$

with $f_{j} \rightarrow f$ in $C\left([-T, T], H^{s}\left(\mathbf{T}^{N}\right)\right)$. The argument above also produces solutions $V_{j}$ to (21) with $g, f$ replaced by $g_{j}, f_{j}$ with $V_{j} \in C\left([-T, T], H^{s+2}\left(\mathbf{T}^{N}\right)\right)$. Since $V_{j}^{\prime}=K V_{j}+f_{j}$, it follows that $V_{j} \in C^{1}\left([-T, T], H^{s+1}\left(\mathbf{T}^{N}\right)\right)$. Hence we can apply the energy inequality (26) and conclude that $\left\{V_{j}\right\}$ is a Cauchy sequence in $C\left([-T, T], H^{s}\left(\mathbf{T}^{N}\right)\right)$. The limit $V$ solves our system. As for uniqueness, since any $V \in C\left([-T, T], H^{s}\left(\mathbf{T}^{N}\right)\right)$, solving (21) must belong to $C^{1}\left([-T, T], H^{s-1}\left(\mathbf{T}^{N}\right)\right) \subset$ $H^{1}\left([-T, T], H^{s-1}\left(\mathbf{T}^{N}\right)\right)$, the energy inequality (26) with $s$ replaced by $s-2$ applies for a difference of two solutions and we see that the solution is unique. Q.E.D. (for Proposition 2).

To return to our iterative method (15), we suppose $g \in H^{M+1}\left(\mathbf{T}^{N}\right), U \in$ $C\left([-T, T], H^{M}\left(\mathbf{T}^{N}\right)\right)$, and $\partial U / \partial t \in C\left([-T, T], H^{M-1}\left(\mathbf{T}^{N}\right)\right)$, where $M>N / 2+1$. Then

$$
K\left(t, x, P_{1} U, \ldots, P_{l} U, D_{x}\right) \in O P H^{M} S^{1},
$$

$f\left(t, x, P_{1} U, \ldots, P_{l} U\right) \in C\left([-T, T], H^{M}\left(\mathbf{T}^{N}\right)\right)$, and by Proposition 2 system (15) has a unique solution

$$
V(t, x) \in C\left([-T, T], H^{M}\left(\mathbf{T}^{N}\right)\right),
$$

such that $(\partial / \partial t) V(t, x) \in C\left([-T, T], H^{M-1}\left(\mathbf{T}^{N}\right)\right)$. In order to prove convergence of the iterative method (15), we will construct equations for various derivatives of $V$. Set

$$
V_{0 \alpha}=D_{x}^{\alpha} V, \quad V_{1 \alpha}=\frac{\partial}{\partial t} D_{x}^{\alpha} V, \quad U_{0 \alpha}=D_{x}^{\alpha} U, \quad U_{1 \alpha}=\frac{\partial}{\partial t} D_{x}^{\alpha} U
$$


Applying the chain rule to (15) yields

(29)

$$
\begin{aligned}
& \frac{\partial}{\partial t} V_{0 \alpha}= K\left(t, x, P_{1} U_{00}, \ldots, P_{l} U_{00}, D_{x}\right) V_{0 \alpha} \\
&+ \sum_{\substack{\gamma+\sigma+\delta=\alpha, \sigma<\alpha \\
\delta=\delta_{1}^{1}+\cdots+\delta_{\mu_{1}}+\cdots+\delta_{1}^{l}+\cdots+\delta_{\mu_{l}}^{l}}} C_{\sigma \gamma \mu_{1} \ldots \mu_{l} \delta_{1}^{1} \ldots \delta_{\mu_{1}}^{1} \ldots \delta_{1}^{l} \ldots \delta_{\mu_{l}}^{l}} \\
& \cdot P_{1} U_{0 \delta_{1}^{1}} \cdots P_{1} U_{0 \delta_{\mu_{1}}^{1}} \cdots P_{l} U_{0 \delta_{1}^{l}} \cdots P_{l} U_{0 \delta_{\mu_{l}}^{l}} K_{\gamma \mu_{1} \ldots \mu_{l}} V_{0 \sigma} \\
&+\sum_{\substack{\gamma+\delta=\alpha \\
\delta=\delta_{1}^{1}+\cdots+\delta_{\mu_{1}}^{1}+\cdots+\delta_{1}^{l}+\cdots+\delta_{\mu_{l}}^{l}}} C_{\sigma \gamma \mu_{1} \ldots \mu_{l} \delta_{1}^{1} \ldots \delta_{\mu_{1}}^{1} \ldots \delta_{1}^{l} \ldots \delta_{\mu_{l}}^{l}}^{\prime} \\
& \cdot P_{1} U_{0 \delta_{1}^{1}} \cdots P_{1} U_{0 \delta_{\mu_{1}}^{1}} \cdots P_{l} U_{0 \delta_{1}^{l}}^{l} \cdots P_{l} U_{0 \delta_{\mu_{l}}^{l}} f_{\gamma \mu_{1} \ldots \mu_{l}}
\end{aligned}
$$

and,

(30)

$$
\begin{aligned}
& \frac{\partial}{\partial t} V_{1 \beta}=K\left(t, x, P_{1} U_{00}, \ldots, P_{l} U_{00}, D_{x}\right) V_{1 \beta} \\
& +\sum_{i=1}^{l} P_{i} U_{10} K_{i} V_{0 \beta}+K_{t} V_{0 \beta} \\
& +\sum_{\gamma+\delta+\sigma=\beta} C_{\sigma \gamma \mu_{1} \ldots \mu_{l} \delta_{1}^{1} \ldots \delta_{\mu_{1}}^{1} \ldots \delta_{1}^{l} \ldots \delta_{\mu_{l}}^{l}} \\
& \cdot\left[\left(P_{1} U_{1 \delta_{1}^{1}} P_{1} U_{0 \delta_{2}^{1}} \cdots P_{1} U_{0 \delta_{\mu_{1}}^{1}} \cdots P_{l} U_{0 \delta_{1}^{l}} \cdots P_{l} U_{0 \delta_{\mu_{l}}^{l}}\right.\right. \\
& \left.+\cdots+P_{1} U_{0 \delta_{1}^{1}} \cdots P_{l} U_{0 \delta_{\mu_{l} l}^{l-1}} P_{l} U_{1 \delta_{\mu_{l}}^{l}}\right) K_{\gamma \mu_{1} \cdots \mu_{l}} V_{0 \sigma} \\
& +P_{1} U_{0 \delta_{1}^{1}} \cdots P_{l} U_{0 \delta_{\mu_{l}}}\left(\sum_{i=1}^{l} P_{i} U_{10} K_{\gamma \mu_{1} \cdots \mu_{i}+1 \cdots \mu_{l}}+K_{\gamma \mu_{1} \cdots \mu_{l} t}\right) V_{0 \sigma} \\
& \left.+P_{1} U_{0 \delta_{1}^{1}} \cdots P_{l} U_{0 \delta_{\mu_{l}^{l}}} K_{\gamma \mu_{1} \cdots \mu_{l}} V_{1 \sigma}\right] \\
& +\sum_{\gamma+\delta=\beta} C_{\sigma \gamma \mu_{1} \cdots \mu_{l} \delta_{1}^{1} \cdots \delta_{\mu_{1}}^{1} \cdots \delta_{1}^{l} \cdots \delta_{\mu_{l}}^{l}}^{\prime} \\
& \cdot\left[\left(P_{l} U_{1 \delta_{1}^{1}} P_{1} U_{0 \delta_{2}^{1}} \cdots P_{1} U_{0 \delta_{\mu_{1}}^{1}} \cdots P_{l} U_{0 \delta_{1}^{l}} \cdots P_{l} U_{0 \delta_{\mu_{l}}^{l}}\right.\right. \\
& \left.+\cdots+P_{1} U_{0 \delta_{1}^{1}} \cdots P_{l} U_{0 \delta_{l}^{l}-1} P_{l} U_{1 \delta_{\mu_{L}}^{l}}\right) \\
& \cdot f_{\gamma \mu_{1} \cdots \mu_{l}}\left(t, x, P_{1} U, \ldots, P_{l} U\right) \\
& +P_{1} U_{0 \delta_{1}^{1}} \cdots P_{1} U_{0 \delta_{\mu_{1}}^{1}} \cdots P_{l} U_{0 \delta_{1}^{l}} \cdots P_{l} U_{0 \delta_{\mu_{l}}^{l}} \\
& \left.\cdot\left(\sum_{i=1}^{l} P_{i} U_{10} f_{\gamma \mu_{1} \ldots \mu_{i}+1 \ldots \mu_{l}}+f_{\gamma \mu_{1} \ldots \mu_{l} t}\right)\right] .
\end{aligned}
$$


Here

$$
\begin{gathered}
K_{\gamma \mu_{1} \ldots \mu_{l}}=K_{\gamma \mu_{1} \ldots \mu_{l}}\left(t, x, \phi_{1}, \ldots, \phi_{l}\right)=D_{x}^{\gamma} D_{\phi_{1}}^{\mu_{1}} \ldots D_{\phi_{l}}^{\mu_{l}} K\left(t, x, \phi_{1}, \ldots, \phi_{l}\right), \\
K_{i}=K_{i}\left(t, x, \phi_{1}, \ldots, \phi_{l}\right)=D_{\phi_{i}} K\left(t, x, \phi_{1}, \ldots, \phi_{l}\right), \quad i=1, \ldots, l
\end{gathered}
$$

and

$$
K_{\gamma \mu_{1} \ldots \mu_{l} t}=D_{t} K_{\gamma \mu_{1} \ldots \mu_{l}} .
$$

$f_{\gamma \mu_{1} \ldots \mu_{l}}$ and $f_{\gamma \mu_{1} \ldots \mu_{l} t}$ are defined similarly.

Now we replace $U_{j \sigma}$ and $V_{j \sigma}$ by $P_{j \sigma}(\tilde{U})$ and $P_{j \sigma}(\tilde{V})$ respectively where

$$
\begin{aligned}
& \tilde{U}=\left\{U_{0 \alpha}, U_{1 \beta}: 0 \leq|\alpha| \leq M, 0 \leq|\beta| \leq M-1\right\}, \\
& P_{j \sigma}(\tilde{U})=\Lambda^{-(M-j-|\sigma|)} \sum_{|\beta|=M-j} C_{\alpha \beta}\left(x, D_{x}\right) U_{j \beta} .
\end{aligned}
$$

$\tilde{V}$ and $P_{j \sigma}(\tilde{V})$ are defined similarly. After these replacements system (29), (30) for $\tilde{V}$ becomes

$$
\begin{aligned}
\frac{\partial}{\partial t} \tilde{V}= & K\left(t, x, P_{1} P_{00} \tilde{U}, \ldots, P_{l} P_{00} \tilde{U}, D_{x}\right) \tilde{V} \\
& +\Phi\left(t, x, P_{1} P_{00} \tilde{U}, \ldots, P_{l} P_{00} \tilde{U}, \tilde{V}\right)
\end{aligned}
$$

Here

$$
K\left(t, x, P_{1} P_{00} \tilde{U}, \ldots, P_{l} P_{00} \tilde{U}, D_{x}\right) \in O P H^{M} S^{1}
$$

and

$$
\Phi\left(t, x, P_{1} P_{00} \tilde{U}, \ldots, P_{l} P_{00} \tilde{U}, D_{x}\right)
$$

contain terms of order $\leq 0$.

Now since $U, V \in \mathbf{R}^{m n}$, it follows that $\tilde{U}, \tilde{V} \in \mathbf{R}^{K}$, where

$$
K=m n\left\{\left(\begin{array}{c}
M+N \\
N
\end{array}\right)+\left(\begin{array}{c}
M+N-1 \\
N
\end{array}\right)\right\}
$$

and we have the following.

LEMma 3. Assuming $M>N / 2+1$, we find that

$$
\begin{aligned}
\Phi: \mathbf{R} \times \mathbf{T}^{N} \times\left[L^{2}\left(\mathbf{T}^{N}\right)\right]^{2 m n}\left\{\left({ }_{N}^{M+N}\right)+\left({ }^{M+N-1}\right)\right\} \\
\left.\left.\rightarrow\left[L^{2}\left(\mathbf{T}^{N}\right)\right]^{m n\left\{\left({ }_{N}+N\right.\right.}\right)+\left({ }_{N}^{M+N-1}\right)\right\}
\end{aligned}
$$

is a Lipschitz continuous map.

PROOF. Consider the components of $\Phi$ of the form

$$
\psi_{1}=\prod_{i=1}^{l} \prod_{\mu=1}^{\mu_{i}} P_{i} P_{0 \delta_{\mu}} \tilde{U} K_{\gamma \mu_{1} \ldots \mu_{l}}\left(t, x, P_{1} P_{00} \tilde{U}, \ldots, P_{l} P_{00} \tilde{U}, D_{x}\right) P_{0 \sigma} \tilde{V}
$$

and

$$
\psi_{2}=\prod_{i=1}^{l} \prod_{\mu=1}^{\mu_{i}} P_{i} P_{0 \delta_{\mu}^{i}} \tilde{U} f_{\gamma \mu_{1} \ldots \mu_{l}}\left(t, x, P_{1} P_{00} \tilde{U}, \ldots, P_{l} P_{00} \tilde{U}\right)
$$

where

$$
\left|\gamma+\sigma+\sum_{i=1}^{l} \sum_{\mu=1}^{\mu_{i}} \delta_{\mu}^{i}\right| \leq M, \quad|\sigma|<M .
$$


The map $\tilde{U} \rightarrow K_{\gamma \mu_{1} \ldots \mu_{l}}\left(t, x, P_{1} P_{00} \tilde{U}, \ldots, P_{l} P_{00} \tilde{U}, D_{x}\right)$ is a Lipschitz map of $L^{2}\left(\mathbf{T}^{N}\right)$ into $O P H^{M} S^{1}$ since $K_{\gamma \mu_{1} \ldots \mu_{l}}\left(t, x, P_{1} P_{00} \tilde{U}, \ldots, P_{l} P_{00} \tilde{U}, D_{x}\right)$ is a $C^{\infty}$ function of its arguments. Consequently, according to (23),

$$
K_{\gamma \mu_{1} \ldots \mu_{l}}\left(t, x, P_{1} P_{00} \tilde{U}, \ldots, P_{l} P_{00} \tilde{U}, D_{x}\right): H^{s}\left(\mathbf{T}^{N}\right) \rightarrow H^{s-1}\left(\mathbf{T}^{N}\right)
$$

for any $s \in \mathbf{R}$. Since $P_{0 \sigma} \tilde{V} \in H^{M-|\sigma|}\left(\mathbf{T}^{N}\right)$ and $P_{0 \delta_{\mu}^{i}} \tilde{U} \in M^{M-\left|\delta_{\mu}^{i}\right|}\left(\mathbf{T}^{N}\right)$, we conclude that $\psi_{1} \in L^{2}\left(\mathbf{T}^{N}\right)$ and, being multilinear, is a Lipschitz function of its arguments. Similarly, the map $\tilde{U} \rightarrow f\left(t, x, P_{1} P_{00} \tilde{U}, \ldots, P_{l} P_{00} \tilde{U}\right)$ is a Lipschitz map of $L^{2}\left(\mathbf{T}^{N}\right)$ into itself since $f\left(t, x, P_{1} P_{00} \tilde{U}(x), \ldots, P_{l} P_{00} \tilde{U}(x)\right) \in H^{M}\left(\mathbf{T}^{N}\right) \subset$ $L^{2}\left(\mathbf{T}^{N}\right)$. Hence, $\psi_{2} \in L^{2}\left(\mathbf{T}^{N}\right)$ and is a Lipschitz function of its arguments. A similar argument controls all the other terms of $\Phi$. Q.E.D.

Next, we construct a positive definite symmetrizer $R\left(t, x, w_{1}, \ldots, w_{l}, D_{x}\right)$ for $K\left(t, x, w_{1}, \ldots, w_{l}, D_{x}\right)$ and substitute $P_{i} P_{00} \tilde{U}$ for $w_{i}, i=1, \ldots, l$, which gives us $R\left(t, x, P_{1} P_{00} \tilde{U}, \ldots, P_{l} P_{00} \tilde{U}, D_{x}\right) \in O P H^{M} S^{0}$. Now we can write

$$
\begin{aligned}
\frac{d}{d t}(R \tilde{V}, \tilde{V})= & (R(K \tilde{V}+\Phi), \tilde{V})+(R \tilde{V}, K \tilde{V}+\Phi)+\left(\left(\frac{\partial}{\partial t} R\right) \tilde{V}, \tilde{V}\right) \\
= & \left(\left(R K+K^{*} R\right) \tilde{V}, \tilde{V}\right)+(R \Phi, \tilde{V})+(R \tilde{V}, \Phi) \\
& +\left(\left(R_{t}+\sum_{i=1}^{l} P_{i} P_{10} \tilde{U} R_{w_{i}}\right) \tilde{V}, \tilde{V}\right) .
\end{aligned}
$$

By the argument (23) the last three terms are bounded by

$$
B\left(\|\tilde{U}\|_{L^{2}}\right)\|\tilde{V}\|_{L^{2}}^{2}+B\left(\|\tilde{U}\|_{L^{2}}\right)
$$

for $t \in[-T, T]$, where $B$ is some function of its arguments. By the same argument and since

$$
\tilde{U} \rightarrow\left(R K+(R K)^{*}\right)\left(t, x, P_{1} P_{00} \tilde{U}, \ldots, P_{l} P_{00} \tilde{U}, D_{x}\right)
$$

is a Lipschitz map of $L^{2}\left(\mathbf{T}^{N}\right)$ into $O P H^{L} S^{0}$ for some $L<M$, we obtain the bound

$$
\left(\left(R K+K^{*} R\right) \tilde{V}, \tilde{V}\right) \leq B\left(\|\tilde{U}\|_{L^{2}}\right)\|\tilde{V}\|_{L^{2}}^{2} .
$$

Consequently, (34) becomes

$$
\frac{d}{d t}(R \tilde{V}, \tilde{V}) \leq B^{\prime}\left(\|\tilde{U}\|_{L^{2}}\right)(R \tilde{V}, \tilde{V})+B^{\prime}\left(\|\tilde{U}\|_{L^{2}}\right)
$$

and Gronwall's inequality yields

$$
\|\tilde{V}(t)\|_{L^{2}}^{2} \leq e^{\int_{0}^{t} B^{\prime}\left(\|\tilde{U}(\tau)\|_{L^{2}}\right) d \tau}\left[\|\tilde{V}(0)\|_{L^{2}}^{2}+\int_{0}^{t} B^{\prime}\left(\|\tilde{U}(\tau)\|_{L^{2}}\right) d \tau\right]
$$

where $\tilde{V}(0)$ is defined similar to $(31)$ with

$$
V_{0 \alpha}(0)=D_{x}^{\alpha} g, \quad V_{1 \beta}(0)=D_{x}^{\beta}(K(t, g) g)+D_{x}^{\beta} f, \quad|\alpha| \leq M,|\beta| \leq M-1 .
$$

Note that $V_{0 \alpha}(0) \in L^{2}\left(\mathbf{T}^{N}\right)$ since $g \in H^{M}\left(\mathbf{T}^{N}\right)$; and $V_{1 \beta}(0) \in L^{2}\left(\mathbf{T}^{N}\right)$ since $f \in H^{M}\left(\mathbf{T}^{N}\right)$ and by the argument used in the proof of Lemma 3. So, we can rewrite (35) as

$$
\|\tilde{V}(\tau)\|_{L^{2}} \leq e^{\int_{0}^{t} B^{\prime}\left(\|\tilde{U}(\tau)\|_{L^{2}}\right) d \tau}\left[C_{0}^{2}\|g\|_{H^{M}}^{2}+\int_{0}^{t} B^{\prime}\left(\|\tilde{U}(t)\|_{L^{2}}\right) d \tau\right] .
$$


Since by assumption $U \in C\left([-T, T], H^{M}\left(\mathbf{T}^{N}\right)\right) \cap C^{1}\left([-T, T], H^{M-1}\left(\mathbf{T}^{N}\right)\right)$, then $\tilde{U} \in C\left([-T, T], L^{2}\left(\mathbf{T}^{N}\right)\right)$. Suppose the norm of $\tilde{U}$ in this space is $\leq A_{0}$ where we pick $A_{0} \geq 2 C_{0}\|g\|_{H^{M}}+1$. Pick the $t$ interval fairly small, as follows. Suppose $T \leq T_{0}$ where $T_{0}$ is so small that, with $B_{1}=\sup \left\{\left|B^{\prime}(\lambda)\right|:|\lambda| \leq A_{0}\right\}$,

$$
e^{T_{0} B_{1}}\left[C_{0}^{2}\|g\|_{H^{M}}^{2}+T_{0} B_{1}\right] \leq A_{0}^{2} .
$$

It follows from (36) that, under this assumption, $\|\tilde{V}\|_{L^{2}} \leq A_{0},|t| \leq T_{0}$. Consequently, for such a small $t$ interval, the mapping $\tilde{V}=\tilde{F} \tilde{U}$ arising from (15) maps the set

into itself.

$$
\left\{\tilde{U} \in C\left([-T, T], L^{2}\left(\mathbf{T}^{N}\right)\right):\|\tilde{U}\|_{L^{2}} \leq A_{0}\right\}
$$

To check the convergence of $V_{K}=F^{K} U$, we need to estimate the difference between $\tilde{V}=\tilde{F}(\tilde{U})$ and $\tilde{V}_{1}=\tilde{F}\left(\tilde{U}_{1}\right)$. From (32) we get

$$
\begin{aligned}
\frac{\partial}{\partial t}\left(\tilde{V}-\tilde{V}_{1}\right)= & K\left(t, x, P_{1} P_{00} \tilde{U}, \ldots, P_{l} P_{00} \tilde{U}, D_{x}\right) \tilde{V} \\
& -K\left(t, x, P_{1} P_{00} \tilde{U}_{1}, \ldots, P_{l} P_{00} \tilde{U}_{1}, D_{x}\right) \tilde{V}_{1} \\
& +\Phi(t, x, \tilde{U}, \tilde{V})-\Phi\left(t, x, \tilde{U}_{1}, \tilde{V}_{1}\right) \\
= & K\left(t, x, P_{1} P_{00} \tilde{U}, \ldots, P_{l} P_{00} \tilde{U}, D_{x}\right)\left(\tilde{V}-\tilde{V}_{1}\right) \\
& +\left[K\left(t, x, P_{i} P_{00} \tilde{U}, D_{x}\right)-K\left(t, x, P_{i} P_{00} \tilde{U}_{1}, D_{x}\right)\right] \tilde{V}_{1} \\
& +\Phi(t, x, \tilde{U}, \tilde{V})-\Phi\left(t, x, \tilde{U}_{1}, \tilde{V}_{1}\right) \\
= & K\left(t, x, P_{i} P_{00} \tilde{U}, D_{x}\right)\left(\tilde{V}-\tilde{V}_{1}\right)+\Delta
\end{aligned}
$$

where, with the aid of Lemma 3 , we have

(39)

$\|\Delta\|_{L^{2}\left(\mathbf{T}^{N}\right)} \leq C\left\|\tilde{U}-\tilde{U}_{1}\right\|_{L^{2}\left(\mathbf{T}^{N}\right)}\left[\left\|\tilde{V}_{1}\right\|_{H^{1}\left(\mathbf{T}^{N}\right)}+C_{1}\right]+C_{2}\left[\|\tilde{V}\|_{L^{2}\left(\mathbf{T}^{N}\right)}+\left\|\tilde{V}_{1}\right\|_{L^{2}\left(\mathbf{T}^{N}\right)}\right]$.

Now, if we set $\hat{U}=\left\{U_{0 \alpha}, U_{1 \beta}:|\alpha| \leq M+1,|\beta| \leq M\right\}$ and define $\hat{V}$ similarly, (15) gives rise to $\hat{V}=\hat{F} \hat{U}$ and the proof of (35) extends to

$$
\|\hat{V}(t)\|_{L^{2}} \leq e^{\int_{0}^{t} B^{\prime}\left(\|\hat{U}(\tau)\|_{L^{2}}\right) d \tau}\left[C_{1}^{2}\|g\|_{H^{M+1}}^{2}+\int_{0}^{t} B^{\prime}\left(\|\hat{U}(\tau)\|_{L^{2}}\right) d \tau\right] .
$$

Pick $A_{1} \geq 2 C_{1}\|g\|_{H^{M+1}}+1$, supposing $g \in H^{M+1}\left(\mathbf{T}^{N}\right)$. Let $T_{1}$ be such that if $\|\hat{U}(t)\|_{L^{2}} \leq A_{1}$ for $|t| \leq T_{1}$, then

$$
\|\hat{V}(t)\|_{L^{2}} \leq A_{1}, \quad|t| \leq T_{1}
$$

This implies

$$
\|\tilde{V}(t)\|_{H^{1}} \leq C A_{1}, \quad|t| \leq T_{1}
$$

and furthermore $\tilde{F}^{\nu} \tilde{U}$ satisfies the estimate (41) for $\nu=1,2,3, \ldots$. Now (38), (39), and (41) yield

$$
\begin{aligned}
\left\|\tilde{V}(t)-\tilde{V}_{1}(t)\right\|_{L^{2}} & \leq A_{2} \int_{0}^{t}|| \tilde{U}(\tau)-\tilde{U}_{1}(\tau) \|_{L^{2}} d \tau, \quad|t| \leq T_{1} \\
& \leq A_{2} T_{1} \sup _{|\tau| \leq T_{1}}\left\|\tilde{U}(\tau)-\tilde{U}_{1}(\tau)\right\|_{L^{2}} .
\end{aligned}
$$


It follows from equation (42) that $\tilde{F}^{\nu} \tilde{U}$ will converge to a limit as $\nu \rightarrow \infty$, in $C\left(\left[-T_{0}, T_{0}\right], L^{2}\left(\mathbf{T}^{N}\right)\right)$, provided $T_{0} \leq \min \left(T, T_{1}\right)$ and $A_{2} T_{0}<1$. The limit, $\tilde{w}$, must be of the form $\left\{w_{0 \alpha}, w_{1 \beta}:|\alpha| \leq M,|\beta| \leq M-1\right\}$ for some

$$
w \in C\left(\left[-T_{0}, T_{0}\right], H^{M}\left(\mathbf{T}^{N}\right)\right) \cap C^{1}\left(\left[-T_{0}, T_{0}\right], H^{M-1}\left(\mathbf{T}^{N}\right)\right)
$$

and $w$ must solve (14). Since the terms $\hat{F}^{\nu} \hat{U}$ are bounded in $L^{\infty}\left(\left[-T_{0}, T_{0}\right], L^{2}\left(\mathbf{T}^{N}\right)\right)$, we can see that $\hat{w} \in L^{\infty}\left(\left[-T_{0}, T_{0}\right], L^{2}\left(\mathbf{T}^{N}\right)\right)$, i.e.

$$
w \in L^{\infty}\left(\left[-T_{0}, T_{0}\right], H^{M+1}(\mathbf{T})\right)
$$

and

$$
\partial w / \partial t \in L^{\infty}\left(\left[-T_{0}, T_{0}\right], H^{M}\left(\mathbf{T}^{N}\right)\right) .
$$

To prove uniqueness, let $w_{1}$ be another solution to (14) with similar regularity. Then (42) implies

$$
\left\|\tilde{w}(t)-\tilde{w}_{1}(t)\right\|_{L^{2}} \leq A_{2} \int_{0}^{t}\left\|\tilde{w}(\tau)-\tilde{w}_{1}(\tau)\right\|_{L^{2}} d \tau
$$

which immediately gives us $\tilde{w}(t)=\tilde{w}_{1}(t)$ or $w(t)=w_{1}(t)$.

We summarize the following.

THEOREM 2. Given $M>N / 2+1$, let $g \in H^{M+1}\left(\mathbf{T}^{N}\right)$. Then for $T$ sufficiently small, the iterative method (15) converges to a unique solution $U$ of (14) with

$$
\begin{aligned}
U & \in C\left([-T, T], H^{M}\left(\mathbf{T}^{N}\right)\right) \cap L^{\infty}\left([-T, T], H^{M+1}\left(\mathbf{T}^{N}\right)\right), \\
\partial U / \partial t & \in C\left([-T, T], H^{M-1}\left(\mathbf{T}^{N}\right)\right) \cap L^{\infty}\left([-T, T], H^{M}\left(\mathbf{T}^{N}\right)\right)
\end{aligned}
$$

provided (14) is either symmetric hyperbolic or strictly hyperbolic.

V. Conclusion. Now we can solve the Cauchy problem (9).

THEOREM 3. System (9), being symmetric hyperbolic or strictly hyperbolic, has a unique solution $U$ on $[-T, T] \times \mathbf{T}^{N}$, provided $M>N / 2+1$ and $T$ small enough, and

$$
\frac{\partial^{j}}{\partial t^{j}} U \in C\left([-T, T], H^{M-j-1}\left(\mathbf{T}^{N}\right)\right) \cap L^{\infty}\left([-T, T], H^{M-j}\left(\mathbf{T}^{N}\right)\right), \quad 0 \leq j \leq m-1 .
$$

Coming back to the original problem (1), we have the following result:

THEOREM 4. Provided $M>N / 2+1$, the Cauchy problem for a system (1) of nonlinear equations with hypotheses (a), (b), (c), (d) has a unique solution $\vec{u}$ on $[-T, T] \times \mathbf{T}^{N}$, such that

$$
\begin{array}{r}
\frac{\partial j}{\partial t^{j}} u_{i} \in C\left([-T, T], H^{M-j}\left(\mathbf{T}^{N}\right)\right) \cap L^{\infty}\left([-T, T], H^{M-j+1}\left(\mathbf{T}^{N}\right)\right), \\
i=1, \ldots, n, j=0, \ldots, m,
\end{array}
$$

if $T$ is small enough. 


\section{REFERENCES}

1. J. Bona and R. Scott, Solutions of the Korteweg-de Vries equation in fractional order Sobolev spaces, Duke Math. J. 43 (1976), 87-99.

2. J. Bony, Calcul symbolique et propagation des singularités les équations aux derivées partielles non linéaires, Ann. Sci. École Norm. Sup. 14 (1981), 209-246.

3. J. Dieudonné, Foundations of modern analyis, Academic Press, New York, 1964.

4. P. Dionne, Sur les problèmes de Cauchy hyperboliques bien posés, J. Anal. Math. 10 (1962-1963), 1-90.

5. R. DiPerna, Singularities of solutions of nonlinear hyperbolic systems of conservation laws, Arch. Rational Mech. Anal. 60 (1976), 75-100.

6. T. Hughes, T. Kato and J. Marsden, Well posed quasilinear second order hyperbolic systems with applications to nonlinear elastodynamics and general relativity, Arch. Rational Mech. Anal. 63 (1976), 273-294.

7. M. Taylor, Pseudodifferential operators, Princeton Univ. Press, Princeton, N.J., 1981.

Department of Mathematics, Rice University, Houston, Texas 77001 